NASA Technical Memorandum 107121

ICOMP-95-27

\title{
On the Application of Chimera/Unstructured Hybrid Grids for Conjugate Heat Transfer
}

Kai-Hsiung Kao

Institute for Computational Mechanics in Propulsion

Lewis Research Center

Cleveland, Ohio

and

Meng-Sing Liou

Lewis Research Center

Cleveland, Ohio

Prepared for the

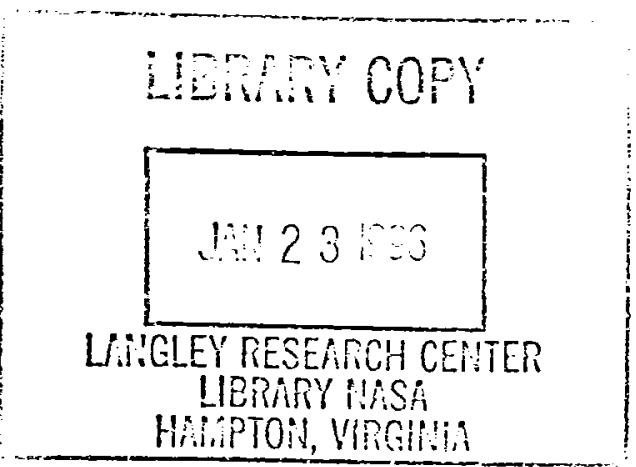

ASME Turbo Expo' 96, Land, Sea, and Air sponsored by the American Society of Mechanical Engineers

Birmingham, England, United Kingdom, June 10-13, 1996

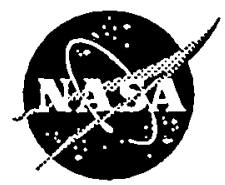

National Aeronautics and Space Administration

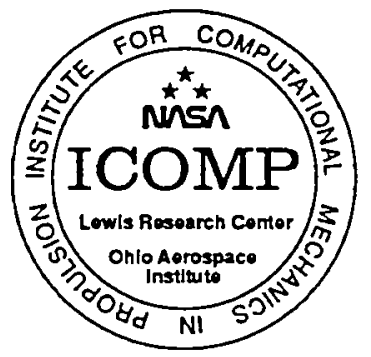


. 


\title{
On the Application of Chimera/Unstructured Hybrid Grids for Conjugate Heat Transfer
}

\author{
Kai-Hsiung Kao \\ Institute for Computational Mechanics in Propulsion \\ NASA Lewis Research Center \\ Cleveland, Ohio 44135 \\ Meng-Sing Liou \\ Internal Fluid Mechanics Division \\ NASA Lewis Research Center \\ Cleveland, Ohio 44135
}

\begin{abstract}
A hybrid grid system that combines the Chimera overset grid scheme and an unstructured grid method is developed to study fluid flow and heat transfer problems. With the proposed method, the solid structural region, in which only the heat conduction is considered, can be easily represented using an unstructured grid method. As for the fluid flow region external to the solid material, the Chimera overset grid scheme has been shown to be very flexible and efficient in resolving complex configurations. The numerical analyses require the flow field solution and material thermal response to be obtained simultaneously. A continuous transfer of temperature and heat flux is specified at the interface, which connects the solid structure and the fluid flow as an integral system. Numerical results are compared with analytical and experimental data for a flat plate and a C3X cooled turbine cascade. A simplified drum-disk system is also simulated to show the effectiveness of this hybrid grid system.
\end{abstract}




\section{Introduction}

Prediction of a turbomachinery engine's performance is a complex process entailing the iterative execution of aerodynamic, thermal, and structural analyses. Ultimately, engine performance is predicted from an aerodynamic analysis of the primary flowpath. However, the accuracy of the aerodynamic analysis is dependent on several factors, one of which is the definition of the flowpath geometry. Accurate determination of the flowpath geometry, which varies depending upon the operation conditions, requires the inclusion of aerodynamic, thermal and structural effects. Consequently, physical situations where heat transfer occurs between material and fluid flow with differing properties are commonly encountered in engineering practice, and often the geometries of interest are extremely irregular.

In recent years, with advancements in performance of computing systems as well as improvements in numerical schemes, computational analyses of the Navier-Stokes equations for fluid flow and heat transfer has become increasingly feasible (Chima, 1985; Ni et al., 1986; Kwon, 1988; Boyle, 1991; Li et al., 1994). Although important progress has been made in solving the Navier-Stokes equations, much work is still needed to achieve robustness, accuracy, and efficiency. An issue which concerns the efficiency and accurancy of the design analysis for engine components is the grid system used for the numerical simulation. For instance, the turbine blades are often characterized by high turning geometry and large mean flow deviation from the axial direction, resulting in substantially skewed grids. The man power required to generate an adequate grid system for various engine components is usually expensive; the procedure is inefficient. Another issue whcih also arises in the analysis is the thermal condition at the solid body surface. In conventional computations, the thermal conditions at the body surface are imposed with either a constant temperature condition or a prescribed heat flux condition when solving the Navier-Stokes equations. In reality, the temperature condition and the heat flux at the solid surface are both unknown a priori and must be determined as a part of the solution. As a result, a conjugate analysis of the fluid flow and heat transfer must be undertaken to obtain physically realistic solutions. 
Among various grid systems for numerical simulation, the Chimera overset grid method (Benek et al., 1985) has been shown to have the most flexibility and efficiency in dealing with very complex configurations (Buning et al., 1989). It consists of a set of overlapped structured grids which are independently generated and body-fitted, yielding a high quality grid readily accessible for efficient solution schemes. Therefore, the Chimera grid method can be utilized to facilitate the grid generation process, resulting in a more efficient design procedure. In the conjugate problem, the fluid flow external to the solid body and the heat conduction inside the solid body must be solved in a coupled fashion. As is known in solid mechanics analysis, most structural problem can be easily resolved by using an unstructured grid method to represent a rigid body. However, any pure-strain approach will not achieve the efficiency for fluid flow and heat transfer problems. Hence, a method that properly employs a hybrid of the Chimera and unstructured grids may prove to be fruitful. A hybrid grid system using an unstructured grid to replace the arbitrarily overlapping region in the framework of the Chimera scheme, termed DRAGON grid, has been developed by Kao and Liou (1995). Their method conserves the numerical fluxes at the grid interface and shows some promising features in terms of the solution accuracy and the grid flexibility. In this paper we adopt their approach by using the unstructured grid to resolve the heat conduction inside the solid body while remaining with the Chimera overset grid scheme for the fluid flow region.

\section{Governing Equations and Numerical Algorithm}

The governing equations for the fluid flow are the 3-D, compressible Navier-Stokes equations. The heat conduction equation is used to evaluate the temperature condition inside a solid material.

\subsection{Navier-Stokes Equations}

The time-dependent compressible Navier-Stokes equations, expressed in an integral form 
over an arbitrary control volume $\Omega$ are:

$$
\int_{\Omega} \frac{\partial \vec{U}}{\partial t} d v+\int_{\partial \Omega} \vec{F} \cdot d \vec{S}=0
$$

where the conservative-variable vector $\vec{U}=(\rho, \rho u, \rho v, \rho w, \rho E)^{\mathrm{T}}$. The inviscid flux is written as a sum of the convective and pressure fluxes: $\vec{F}=\Phi \vec{V}+\vec{P}$, where $\Phi=(\rho, \rho u, \rho v, \rho w, \rho H)^{T}$ is the vector containing convected variables and $\vec{P}=p(0, \dot{i}, \dot{j}, \vec{k}, 0)^{T}$. The specific total energy is $E=e_{i}+|\vec{V}|^{2} / 2=H-p / \rho$. We denote with an overhead arrow the vector quantities expressed in terms of Cartesian coordinates.

Based on the cell-centered finite volume method, the governing equations are semi-discretized. We use the new flux scheme AUSM $^{+}$developed by Liou (1994) to express the numerical flux at the cell faces. The AUSM+ ${ }^{+}$scheme allows an exact capture of a normal shock by using a suitably chosen interface speed of sound, yields a smoother solution by way of including higherorder polynomials, and leads to faster convergence rate.

The semi-discretized form, describing the rate of time change of $\vec{U}$ in $\Omega$ via the balance of fluxes through all enclosing faces, $\vec{S}_{l}, l=1, \ldots, L X$, whether they be in the structured or unstructured grid regions, can be cast as

$$
\int_{\Omega} \frac{\partial \vec{U}}{\partial t} d v+\sum_{l=1}^{L X} \vec{F}_{l} \bullet \vec{S}_{l}=0
$$

The last terms account for only normal components of the flux at the face, in terms of which the equations are written as:

$$
\int_{\Omega} \frac{\partial \vec{U}}{\partial t} d v+\sum_{l=1}^{L X} F_{n l}\left|\vec{S}_{l}\right|=0
$$

where $F_{n l}=\vec{F}_{l} \bullet \vec{n}_{l}$ and $\vec{n}_{l}$ is the unit normal vector of $\vec{S}_{l}$. The task is then to represent the numerical flux at the cell interface $\vec{S}_{l}$, which straddles cells denoted by subscripts "L" and "R". 
The AUSM+scheme gives the numerical flux in the following expression.

$$
F_{n l}=\tilde{M}_{l} \frac{a_{l}}{2}\left(\Phi_{L}+\Phi_{R}\right)-\left|\tilde{M}_{l}\right| \frac{a_{l}}{2} \Delta \Phi+P_{n l}
$$

In this formula, $a_{l}$ is the speed of sound suitably defined at the interface to result in an exact resolution of a normal shock. Here $\tilde{M}_{l}$ is the interface Mach number and $P_{n l}$ is the interface pressure in the surface normal direction.

\subsection{Heat Conduction Equation}

The conductive heat transfer in the solid structure is governed by the integral heat conduction equation,

$$
\int_{\Omega} \frac{\partial E}{\partial t} d v+\int_{\partial \Omega} \vec{q} \cdot d \vec{S}=0
$$

where $E=\rho_{s} C T$ denotes the potential energy of the solid material and $\vec{q}$ is the heat flux,

$$
\vec{q}=-\kappa_{s} \vec{\nabla} T
$$

Here $\kappa_{s}$ is the thermal conductivity of the material, $\rho_{s}$ is the density and $C$ is the specific heat.

The above integral heat equation is then expressed using the finite volume method. The result is that the heat flux through a surface depends on the temperature in the neighboring cells near the surface. The change in temperature in a cell therefore can be obtained by integrating the heat fluxes throughout the surrounding cell faces. To be consistent with the flow solver, this system is solved iteratively, using a two-step predictor/corrector method on each time step.

\subsection{Time Integration}

The time integration scheme updates the conservative variables at the cell center. The present method originates from the Taylor series expansion in time, as was done by the Lax-Wendroff scheme. Then a two-step scheme with second order time accuracy can be obtained. 


\section{Predictor:}

$$
U^{*}=U^{n}+\Delta t \cdot \frac{d}{d t} U^{n}
$$

Corrector:

$$
\begin{aligned}
U^{* *} & =U^{*}+\Delta t \cdot \frac{d}{d t} U^{*} \\
U^{n+1} & =\frac{1}{2}\left(U^{n}+U^{* *}\right)=\frac{1}{2}\left\{U^{*}+\left[U^{n}+\Delta t \cdot \frac{d}{d t} U^{*}\right]\right\}
\end{aligned}
$$

It is noted that (1) the predictor step allows a full time step, and (2) like other 2-step integration schemes, only two levels of storage are needed as $U^{n}$ is absorbed in forming the residual indicated in the squared bracket, (3) both predictor and corrector steps are identical, with no need of defining a midpoint for the corrector step, leading to simplification of coding and the complexity of evaluating the transport terms.

The stability of the scheme is restricted by the CFL (Courant-Friedrichs-Lewy) number not exceeding unity. For steady state cases, local time stepping is used to accelerate convergence.

\section{Grid Methods}

The Chimera composite grid method has been shown to be very flexible and efficient in generating body-fitted structured grids for complex configurations. In the solid mechanics community, unstructured grid techniques are broadly used to represent structural domains. A combination of these two grid systems is developed to tackle the conjugate heat transfer problems in this paper.

\subsection{Chimera Grid}

The Chimera scheme is a grid embedding technique which provides a conceptually simple method for domain decomposition. For instance, a major grid is generated about a main body element and minor grids are then overset on the major grid so as to resolve interesting features of the configuration. Usually the minor grids are overset on top of the major grid without requiring the 
mesh boundaries to join in any apecial way. However, a common or overlap region is always required to provide the means of matching the solution across boundary interfaces. To increase the flexibility in the selection of subdomains, the Chimera scheme also allows a means of removing regions of a mesh containing an embedded grid. That is, an embedded mesh introduces a "hole" into the mesh in which it is embedded. Typically, a hole is defined by a creation boundary which contains a surface or a group of surfaces. The purpose of a hole creation boundary is to identify points that are within this boundary. A mesh point is considered to be inside a hole creation boundary if it is inside all surfaces that define the boundary. Figure 1 illustrates the connections between composite overlapping grids, with hole points being blanked by a prescribed creation boundary. A practical application of the Chimera grid system for the complete Space Shuttle/Solid Rocket Booster geometry (Buning et al., 1989) is shown in Fig. 2.

A flow solver must be modified to account for the use of multiple meshes and the "hole" in the grids. These hole points must be blanked or excluded from the flow field solution. The main change in the flow algorithm itself is the treatment of the hole boundaries. The blanked solutions are updated in the interpolation routine. With this approach, no special routine or logic tests are required to exclude the blanked points from the flow field solution. Details regarding the grid embedding technique can be found in Steger et al. (1987) and Suh et al. (1990).

\subsection{Unstructured Mesh}

The Delaunay triangulation scheme is applied to generate an unstructured grid in the solid material region. The steps for adopting the unstructured cells in the framework of the Chimera grid scheme are summarized below.

1. Boundary nodes provided by the Chimera grid meshes are reordered according to their geometric coordinates (Barth, 1994).

2. The Delaunay triangulation method is then performed to connect these boundary nodes based on the Bowyer algorithm (1981).

3. In the unstructured grid, since their is no logical ordering of the cells and their neighbors, connectivity matrices containing cell-based as well as edge-based information are introduced. 
Also, the present approach requires additional matrices to connect the structured and unstructured grids. The connectivity matrices used in the present $2 \mathrm{D}$ version are summarized as follows.

(a) IEDGENODE(1:2, edges) - 2 nodes for each edge,

(b) ICELLEDGE(1:3, cells) - 3 edges for each triangular cell,

(c) IEDGECELL(1:2, edges) - 2 neighboring cells for each edge,

(d) IEDGETYPE(edges) - edge type (type of boundary condition),

(e) IEDGEFLUX(grids, edges) - edge number that connects structured and unstructured grids, where the parameter "grids" identifies which structured grid,

(f) IFLUXINDX(grids, edges) - $i$-index of the structured cell that shares the edge fluxes with the unstructured cell,

(g) IFLUXINDY(grids, edges) - $j$-index of the structured cell that shares the edge fluxes with the unstructured cell.

The first four types of connectivities are standard in unstructured grid codes, except that we assign "+" and "-" values to IEDGETYPE to indicate whether the outward normal vector of a triangular cell face points into or out of the neighboring structured cell which shares the same face. This information is needed as the tempercture and heat flux conditions are enforced to be identical on this fase.

Questions concerning triangular grid quality and improvements are not pursued for they are beyond the scope of the present paper.

\subsection{Data Communication Through Grid Interfaces}

In the Chimera method, communication between overlaid grids is achieved by interpolation of boundary values from the mesh or meshes in which the boundaries are contained. We have used the PEGSUS codes (Benek et al., 1986; Suhs et al., 1991) to make interconnections of subdomains, define the hole regions, and supply points to facilitate communication among grids during the solution process. At present, the Chimera scheme employs bilinear interpolation that, as some simple experiments have shown (Mastin et al., 1984; Parthasarathy, 1984), is superior to Taylor series expansion. 
In the current work, both the structured and unstructured solver are based on the cell centered scheme in which quadrilateral and triangular cells are used repectively. Figure 3 shows the interfaces connecting both structured and unstructured grids. As noted, the heat flux, evaluated at the cell interface, is based on the conditions of neighboring cells. In the conjugate problem, the temperature and the heat flux values at the interface are continuously transferred according to the following relations:

$$
\begin{gathered}
T_{w}=T_{f}=T_{s} \\
\vec{q}_{w}=-\kappa_{f} \vec{\nabla} T_{f}=-\kappa_{s} \vec{\nabla} T_{s}
\end{gathered}
$$

Here the subscripts $w, f$ and $s$ denote the values at the solid body surface, the fluid boundary and the solid boundary, respectively.

\section{Test Results and Discussions}

Numerical results are compared with analytical and experimental data for a flat plate and a C3X cooled turbine cascade. A simplified drum-disk system is also simulated to show the effectiveness of the hybrid grid method. For the hybrid grids, both Chimera and unstructured grids can be displayed on the same plot using the Flow Analysis Software Toolkit (FAST; Walatka et al., 1993) visualization package on an IRIS workstation.

\section{Case 1 - Conjugate Heat Transfer on a Flat Plate}

The first case is a flat plate with a cylindrical leading edge, as shown in Fig. 4. The upstream flow conditions are $M=0.3, R e_{l}=10^{4}, P r=0.71$ and $T_{\infty}=300^{\circ} \mathrm{K}$. Here $l=1 \mathrm{~m}$ is the chord length of the plate and the wall thickness is $2.5 \mathrm{~cm}$. Aluminum, with a density of $2700 \mathrm{~kg} /$ $\mathrm{m}^{3}$, thermal conductivity of $211 \mathrm{~W} / \mathrm{m}-{ }^{\circ} \mathrm{K}$ and a specific heat of $900 \mathrm{~W}-\mathrm{s} / \mathrm{kg}-{ }^{\circ} \mathrm{K}$, is used as the plate material. The inner wall temperature is set to be $280^{\circ} \mathrm{K}$ throughout the computation.

Under these conditions, the temperature along the outer wall is nearly constant and equal to the inner wall temperature. Therefore, the calculated Nusselt number can be compared with the 
analytical values, which are given for a flat plate of zero thickness and constant wall temperature by Eckert et al. (1941).

$$
N u=0.333 \sqrt[3]{\operatorname{Pr}} \sqrt{\operatorname{Re} x} \cdot \frac{l}{x}
$$

The computed Nusselt number can be obtained by

$$
N u=\frac{q \cdot l}{\kappa_{f}\left(T_{w}-T_{a}\right)}
$$

where $q$ is the heat flux, $l$ is the plate length, $T_{w}$ is the wall temperature, $T_{a}$ is the adiabatic wall temperature, and $\kappa_{f}$ is the thermal conductivity of the fluid.

Figure 5 shows the hybrid grid system used for the flat plate case. The external flow field is first resolved using one structured mesh with grid dimensions of $168 \times 50$. The Delaunay triangulation method is then used to construct the unstructured grid to represent the flat plate. In this case, we use 530 nodes and 800 triangular cells for the unstructured grid region. The computed Nusselt numbers along the plate surface are compared with the analytical values and displayed in Fig. 6. The profiles are nearly identical everywhere except near the leading edge, where the analytical results are not valid.

\section{Case 2 - C3X Cooled Turbine Cascade}

In this case, the C3X cooled turbine cascade reported by Hylton et al. (1983) is simulated for the conjugate heat transfer solution. The blade geometry considered has been shown experimentally to exhibit a two dimensional response. The blade was instrumented with surface thermocouples and pressures sensors. Data on the blade surface were acquired via a computer controlled data acquisition system, and reduced data are tabulated and reported in Hylton et al. (1983).

A C3X test case (run 112) is selected and its set-up conditions are listed in Hylton et al. (1983). The coolant flow conditions and the experimentally determined external blade wall temperature, heat transfer coefficient, and pressure distributions are also described in that report.

At the inlet, for run case 112 , the Mach number is 0.17 , the Reynolds number is $0.53 \times 10^{6}$, 
the total temperature is $783^{\circ} \mathrm{K}$, and the total pressure is $6.22 \mathrm{KPa}$. The blade geometry possesses a high turning angle which causes difficulties in the grid generation process. A Chimera overset grid system, as displayed in Fig. 7, utilizes an O-type grid (size 139x51) wrapping around the turbine cascade, and an H-type grid (size 76x70) to cover the fluid flow path. This grid system can be applied with ease, not only to preserve high grid quality at the region near the blade body, but also to maintain the grid periodicity at the upper and lower boundaries. The Delaunay triangulation method is then used to generate the unstructured grid representing the inner region of the blade, with a grid size of 610 nodes and 1001 triangular cells. Note that the 10 cooling holes are also included to simulate the conjugate heat transfer solution with cooling effect.

The Baldwin-Lomax algebraic turbulence model reported in Baldwin et al. (1978) is used to calculate the eddy viscosity. The effective viscosity is then given by

$$
\mu=\mu_{l}+\mu_{t}=\Upsilon\left(\frac{\mu_{l}}{P r_{l}}+\frac{\mu_{t}}{P r_{t}}\right)
$$

Here the subscripts $l$ and $t$ refer to the values in the laminar and turbulent flows. In the fluid flow and the solid body regions, the thermal conductivities remain unchanged throughout the computations. The Prandtl numbers, $P r_{l}$ and $P r_{t}$, are equal to 0.71 and 0.9 for laminar and turbulent flows, respectively. The solid material used for the blade body is stainless steel.

Two types of blade boundary conditions are applied in this test case: (1) adiabatic wall, and (2) conjugate blade surface with internal cooling effect. Figures 8-9 illustrate the Mach number contours and the temperature distributions for the adiabatic and conjugate cases, respectively. The pressure distributions along the blade surface for both adiabatic wall and conjugate wall cases, as shown in Fig. 10, indicate close agreement with the experimental data.

As the results plotted in Fig. 11 indicate the adiabatic wall temperature is significantly higher than the experimentally determined blade surface temperature. The prediction of the blade temperature is greatly improved when the conjugate heat transfer analysis with internal cooling effect is included. It is seen that the conjugate blade temperature displayed in Fig. 11 agrees well with the experimental data in both the pressure and the suction regions. However, the discrepancy 
shown around the trailing edge of the blade indicates a higher-order turbulence model may be needed to improve the solution of the wake flow.

\section{Case 3 - Simplified Drum-Disk System}

A secondary flow system in the gas turbine engine is an example which includes aero, thermal and structural analyses. In this test case, a simplified drum-disk flow system is simulated using the present hybrid grid method. Rotating effects of the drum-disk system are not considered in the present calculation.

As illustrated in Fig. 12, the Chimera overset grid scheme is used to represent the interior region between the drum and the disk. It is noted that the disk grid $(88 \times 21)$ is embedded on the drum grid $(116 \times 61)$. Consequently, those grid points inside a prescribed hole creation boundary have been blanked out from the drum grid. As also displayed in Fig. 12, an unstructured grid has been constructed to fill in the disk body, with a grid size of 195 nodes and 301 triangular cells.

In this test case, two types of thermal boundary conditions are used at the disk-fluid conjugate surface, namely the adiabatic wall and conjugate heat transfer wall conditions. Figures 13 and 14 illustrate the pressure and Mach number contours, respectively. It is apparent from the plots that the effect of heat transfer has little influence on the fluid flow solution.

The computed temperature distributions for the drum-disk system are laso displayed in Fig. 15 , indicating similarities in most contours in the fluid region except that some major differences are found near the disk surface. Comparisons of the temperature distributions along the disk body surface are ploted in Fig. 16, showing the average adiabatic wall temperature is much higher than the conjugate wall temperature. Subject to the adiabatic wall assumption, the heat flux is mainly convected by the fluid flow and its value vanishes at disk body surface, causing a large temperature deviation. With the conjugate wall condition, the heat flux is conducted as well as convected within the fluid flow and solid body regions, promising a better thermal exchange at the disk surface. Note that the temperature variations along the surface of the disk body are rather small, and this trend is consistent with the design condition in the secondary flow system. 


\section{Conclusions}

In this paper we perform computations for conjugate heat transfer problems using a hybrid grid system. The fluid flow and solid structural regions are resolved by the Chimera overset grid scheme and unstructured grid method, respectively. In the computational process, the temperature and the heat flux at the cell interfaces between the fluid and solid regions are enforced to be continuously transferred, with no artificial temperature distribution or prescribed heat transfer rate specified. The effects of heat conduction in the solid regions are shown to significantly affect the computed surface temperatures in comparison to those obtained from a traditional adiabatic wall assumption. With the proposed hybrid grid system, aerodynamic analysis for designing the engine components can now efficiently include aero, thermal and structural effects as one integral system.

\section{Acknownledgements}

The computational results for the test problems were obtained using the CRAY-YMP computer system at NASA Lewis Research Center and partly calculated on the C90 at NASA Ames Research Center.

\section{References}

Baldwin, B. S. and Lomax, H., (1978), "Thin layer approximation and algebraic model for separated turbulent flows," AIAA Paper 78-20776.

Barth, T. J., (1994), “Aspects of unstructured grids and finite-volume solvers for the Euler and Navier-Stokes equations," von Karman Institute for Fluid Dynamics, Lecture Series 1994-05.

Benek, J. A., Buning, P. G. and Steger, J. L., (1985), “A 3-D Chimera grid embedding technique," AIAA Paper 85-1523.

Benek, J. A., Steger, J. L., Dougherty, F. C. and Buning, P. G., (1986), “Chimera: A gridembedding technique," AEDC-TR-85-64.

Bowyer, A., (1981), “Computing Dirichlet tessellations," The Computer J., Vol. 24, No. 2, 
pp. 162-166.

Boyle, R. J., (1991), “Navier-Stokes analysis of turbine blade heat transfer," J. Turbomachinery, Vol. 113, pp. 392-403.

Buning, P. G., Chiu, I. T., Martin, F. W. Jr., Meakin, R. L., Obayashi, S., Rizk, Y. M., Steger, J. L. and Yarrow, M., (1989), "Flowfield simulation of the Space Shuttle vehicle in ascent," Proceedings of the Fourth Internation Conference on Supercomputing, Santa Clara, CA.

Chima, R. V., (1985), "Inviscid and viscous flows in cascade with an explicit mutli-grid algorithm," AIAA Journal, Vol. 23, pp. 1556-1563.

Eckert, E. and Weise, W., (1941), "Die temperature unbeheizter korper in einem gasstrom hoher geschwindigkeit," Forschg. Ing.-Wes., Vol. 12, pp. 40-50.

Hylton, L. D., Mihelc, M. S., Turner, E. R., Nealy, D. A. and York, R. F., (1983), “Analytical and experimental evaluation of the heat transfer distribution over the surface of turbine vanes," NASA CR 168015.

Kao, K. H. and Liou, M. S., (1995), "Advance in overset grid schemes: from Chimera to DRAGON grids," AIAA J., Vol. 33, No. 10, pp. 1809-1815.

Kwon, O. K., (1988), "Navier-Stokes solution for steady two-dimensional transonic cascade flows," ASME J. Turbomachinery, Vol. 110, pp. 339-346.

Li, H. and Kassab, A. J., (1994), "Numerical prediction of fluid flow and heat transfer in turbine blades with internal cooling," AIAA Paper 94-2933.

Liou, M. S., (1994), “A continuing search for a near-perfect numerical flux scheme; Part I: AUSM+," NASA TM 106524.

Mastin, C. W. and McConnaughey, H. V., (1984), "Computational problems on composite grids," AIAA Paper 84-1611.

Ni, R. H., Davis, R. L. and Carter, J. E., (1986), “Cascade viscous flow analysis using the Navier-Stokes equations," AIAA Paper 86-0033.

Parthasarathy, K. N., (1984), "Numerical procedure for aircraft/store flow field," Quarterly Progress Reports Nos. 4, 5, and 6, NASA Contract NAS2-11742, General Dynamics, Fort Worth. 
Steger, J. L. and Benek, J. A., (1987), "On the use of composite grid schemes in computational aerodynamics," Comp. Methods Appl. Mech. and Eng., Vol. 64, Nos. 1-3.

Suhs, N. E. and Tramel, R. W., (1991), "PEGSUS 4.0 user's manual," Calspan Corporation/ AEDC Operations, AEDC-TR-91-8.

Walatka, P. P., Clucas, J., McCabe, R. K., Plessel, T. and Potter, R., (1993), "FAST User Guide," NASA Ames Research Center, RND-93-010. 


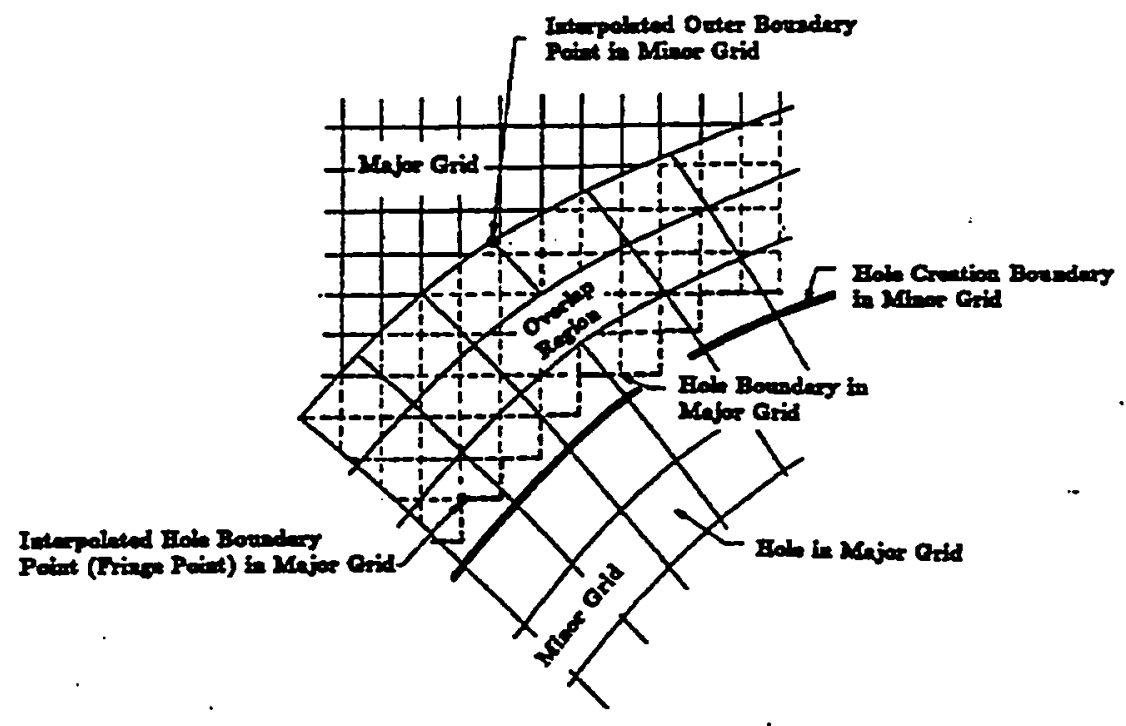

Fig. 1 Interconnections of minor and major grids for Chimera overset scheme.

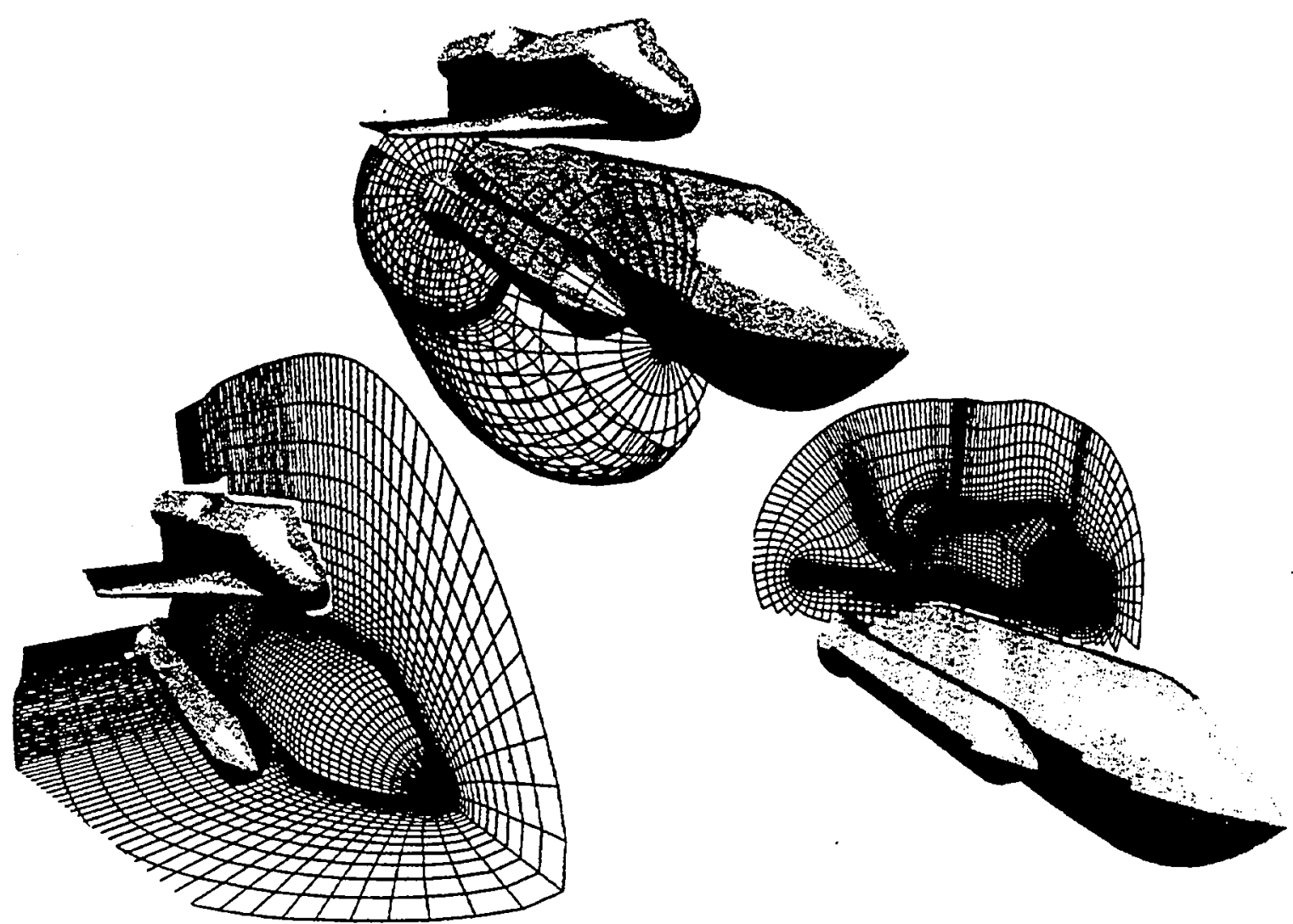

Fig. 2 Chimera grid used for integrated Space Shuttle geometry. 


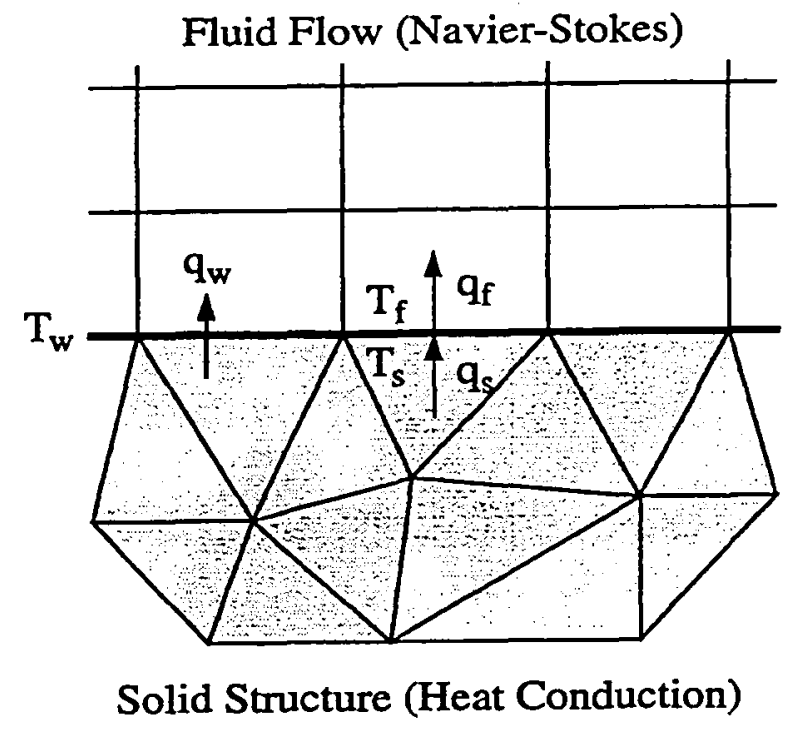

Fig. 3 Heat flux at the cell face connecting the structured and unstructured grids.

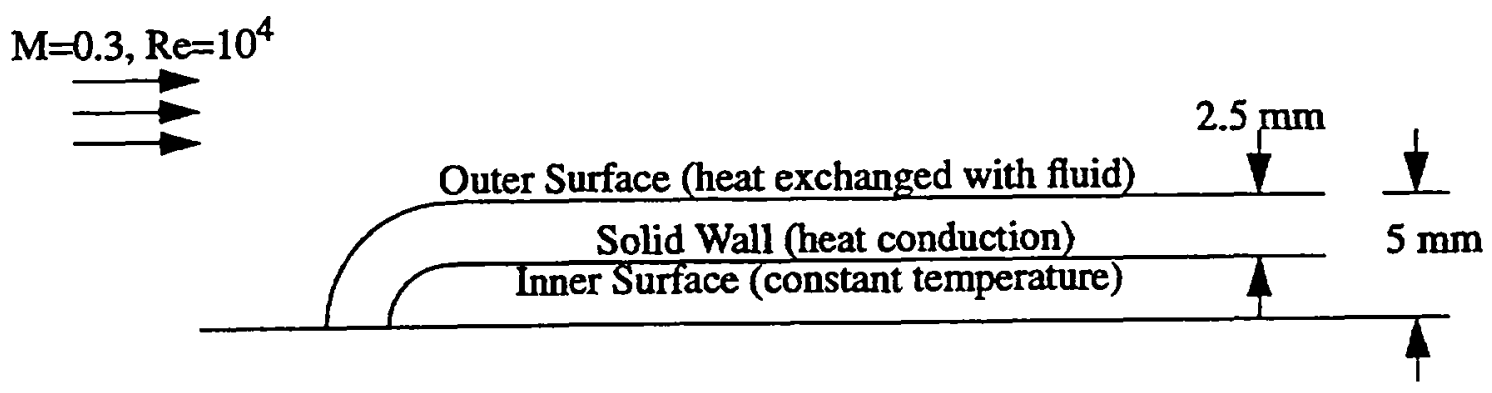

Fig. 4 Conjugate heat transfer on a semi-infinite flat plate. 


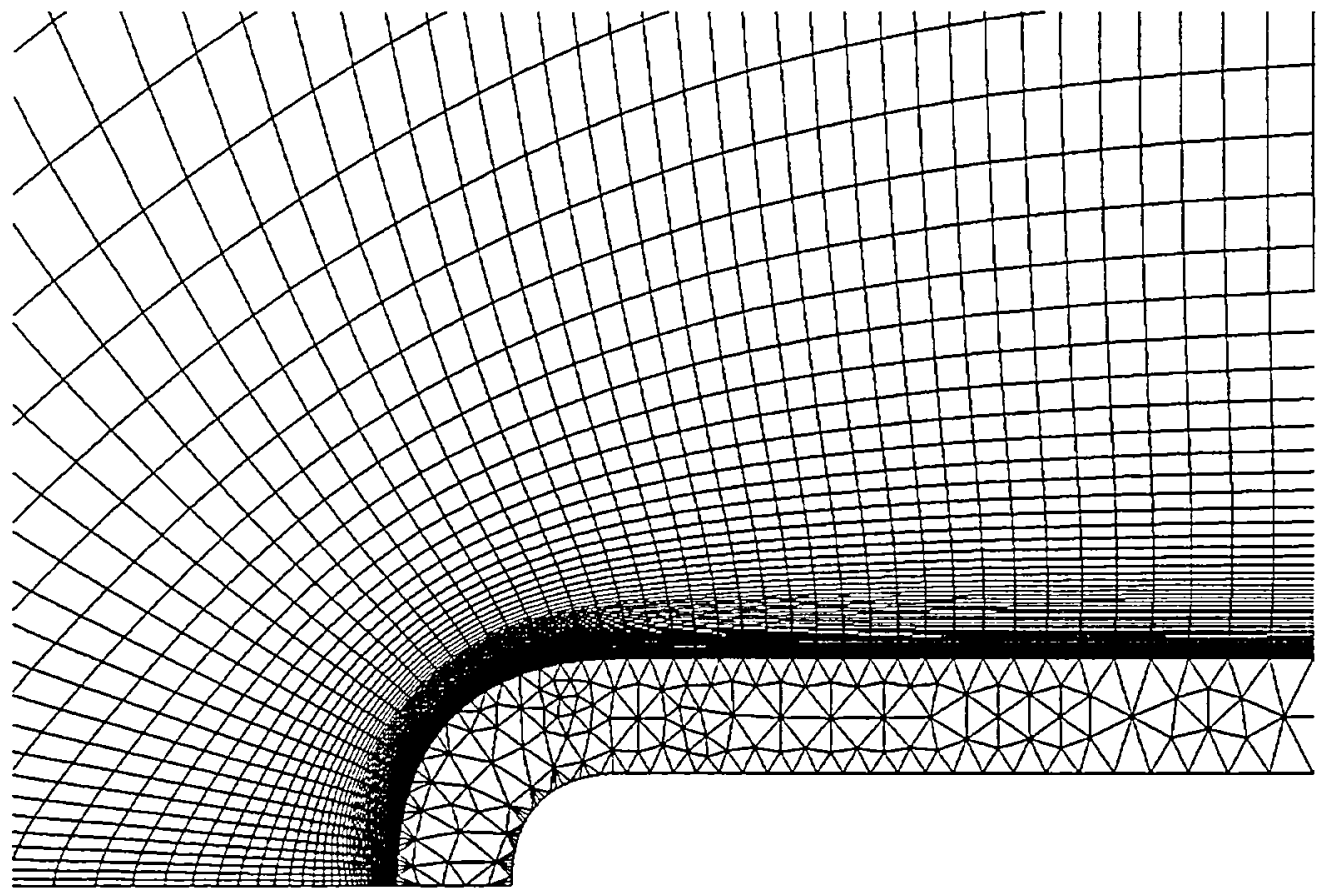

Fig. 5 Hybrid grid system for a cooled flat plate.

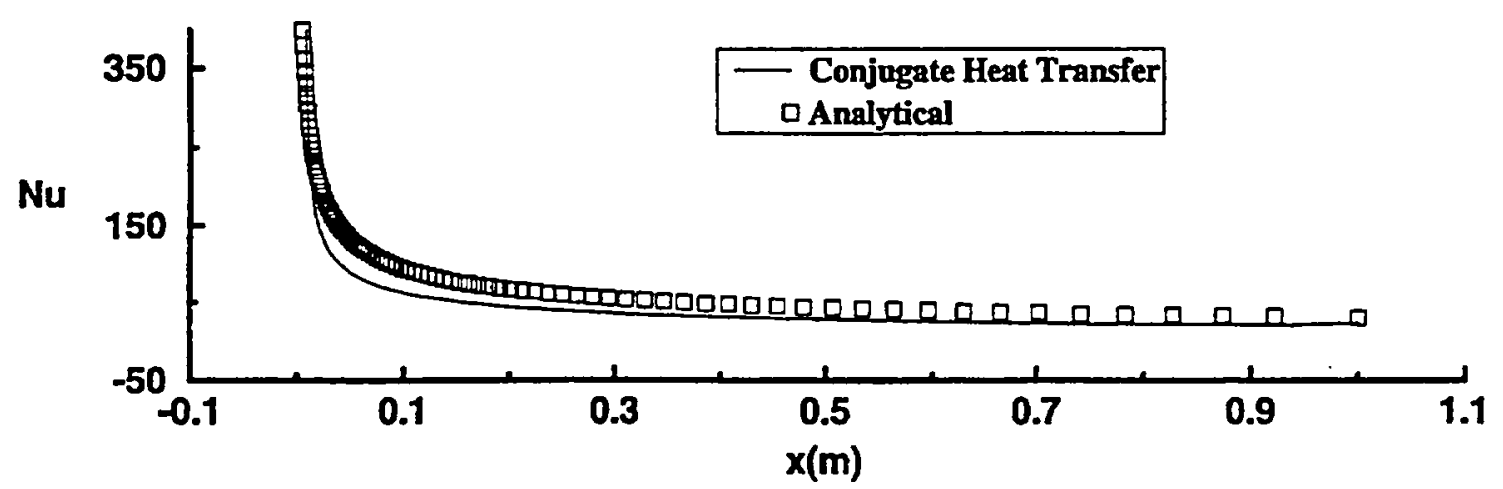

Fig. 6 Comparison of the Nusselt number between conjugated and analytical data. 


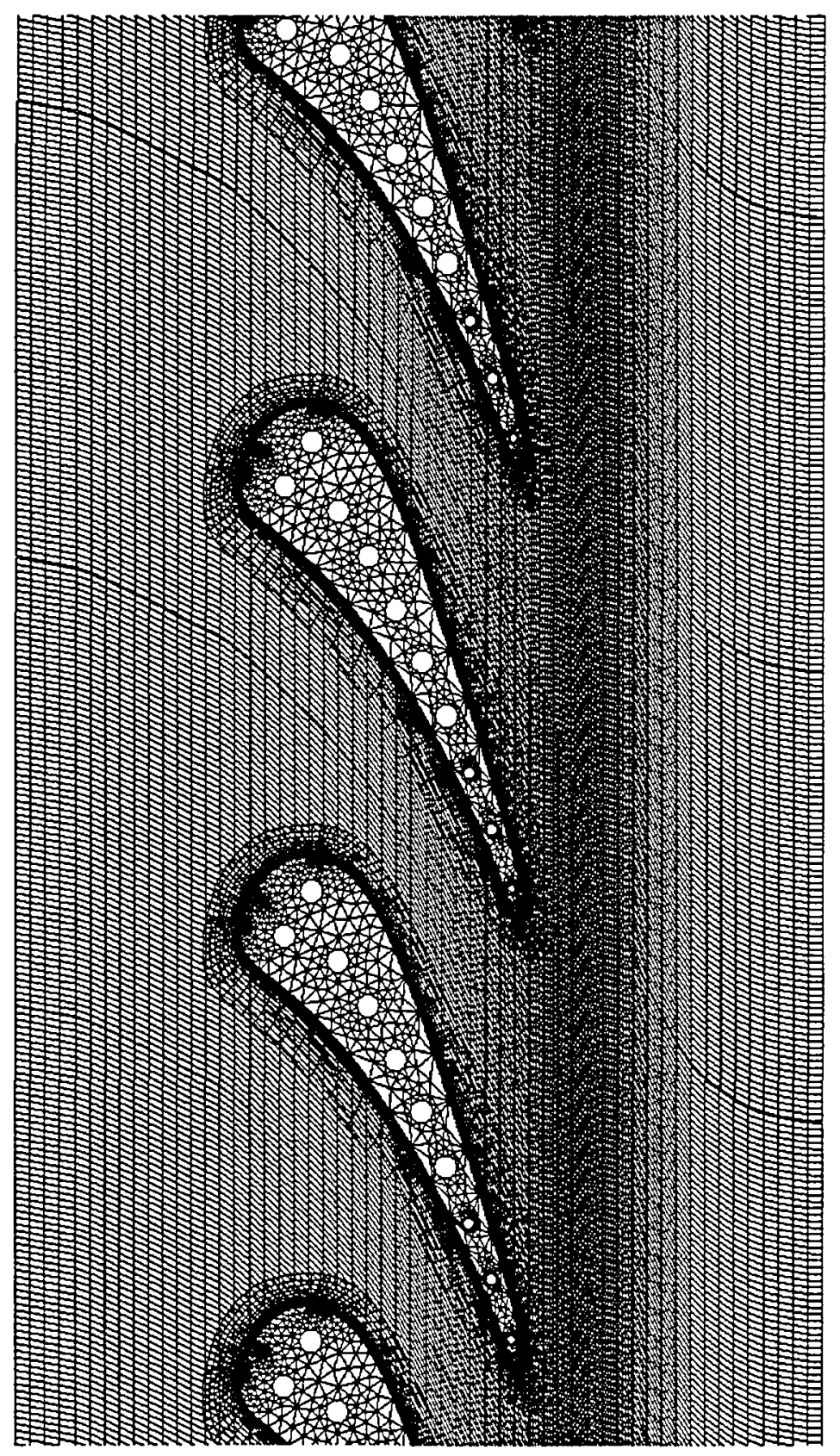

Fig. 7 The Chimera/unstructured grid system used for C3X turbine cascade with 10 cooling holes. 
(a) adiabatic wall

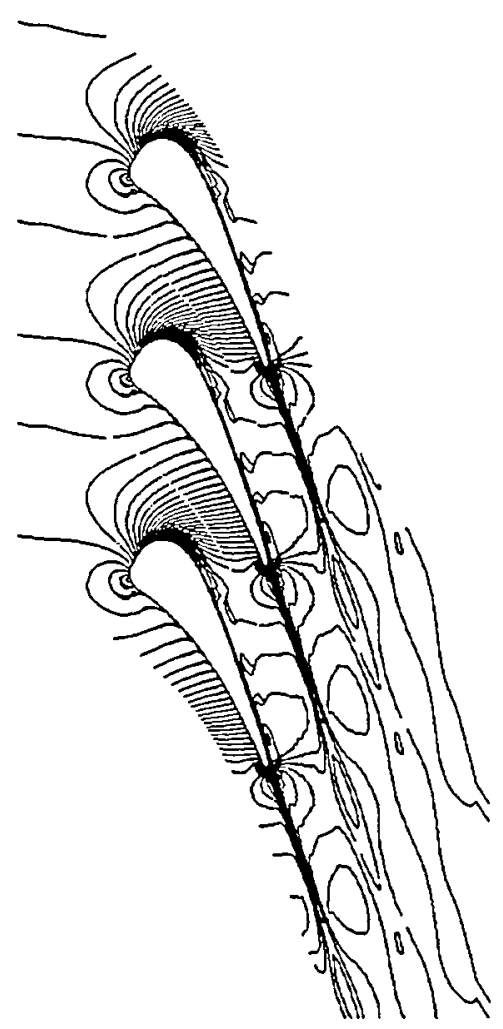

(b) conjugate with cooling

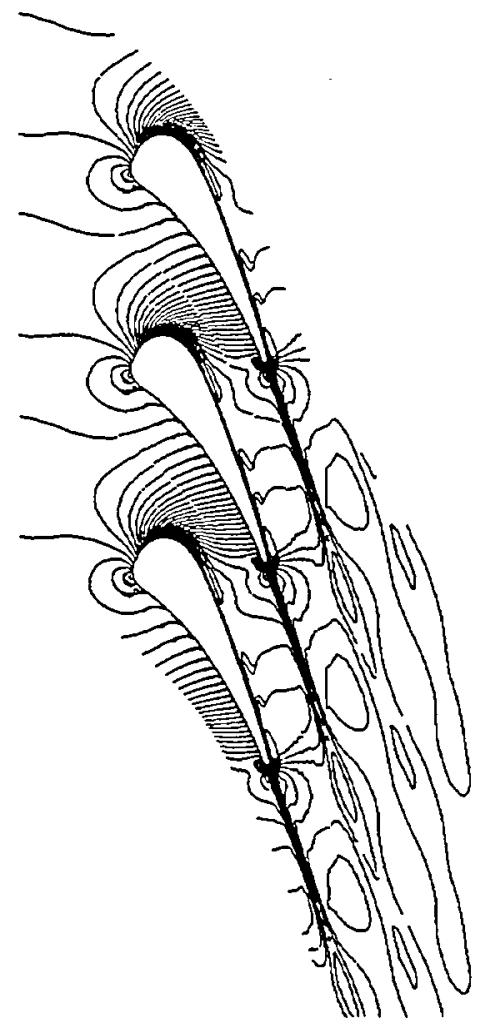

Fig. 8 Mach contours for C3X turbine cascade with adiabatic and conjugate walls.

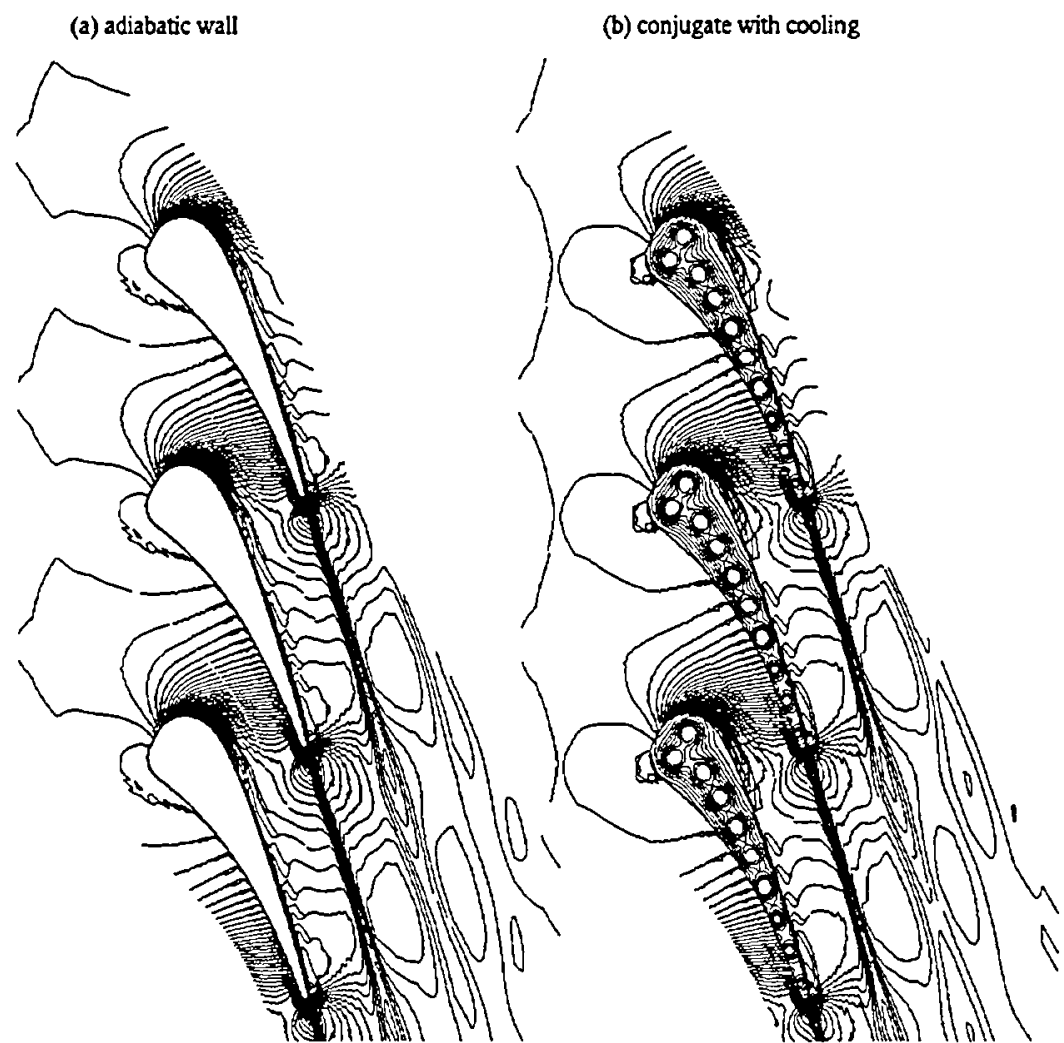

Fig. 9 Temperature distributions for (a) adiabatic and (b) conjugate with cooling walls in the C3X turbine cascade flows. 


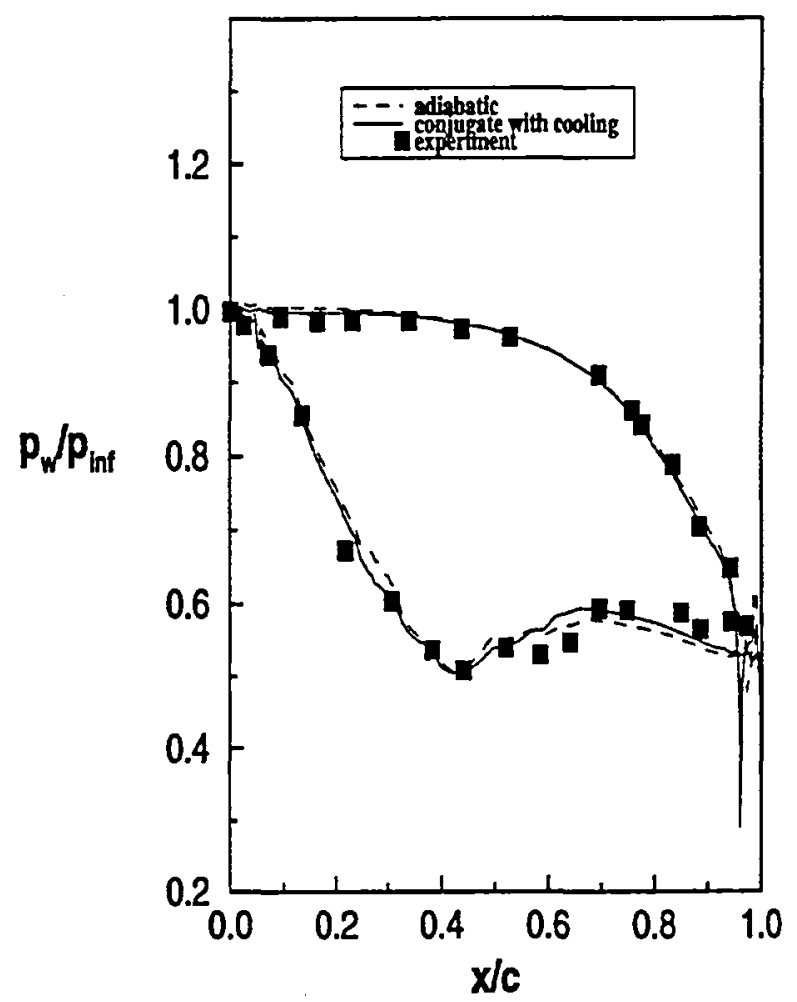

Fig. 10 Pressure distributions along the blade surface.

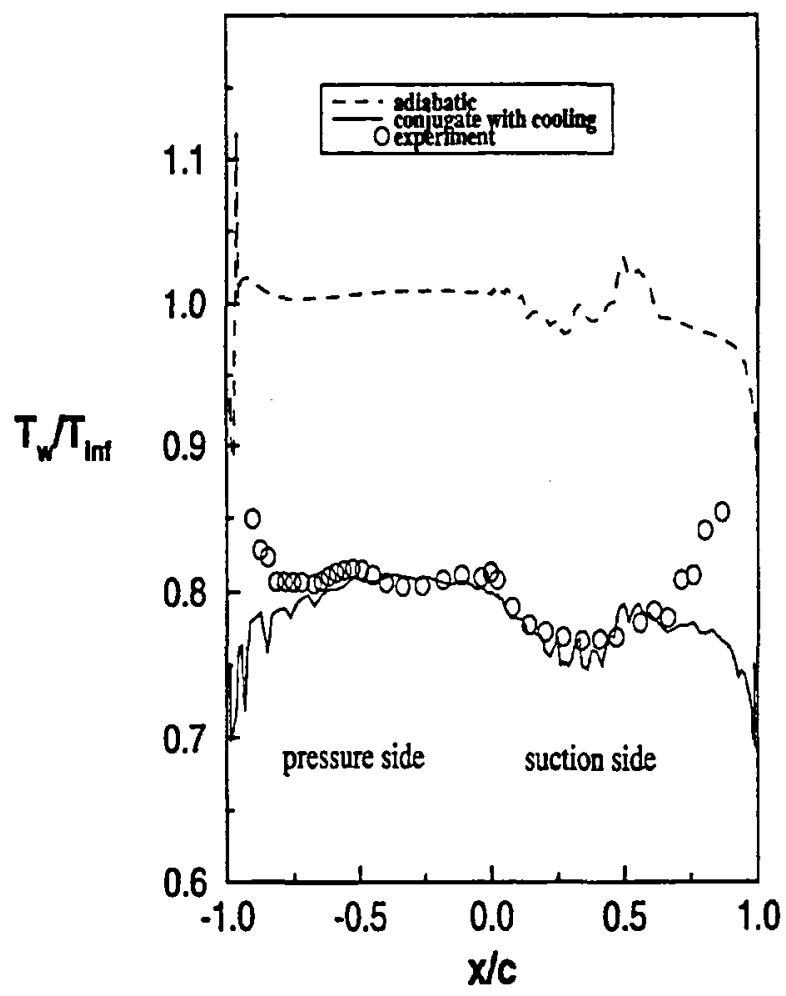

Fig. 11 Temperature distributions along the blade surface. 


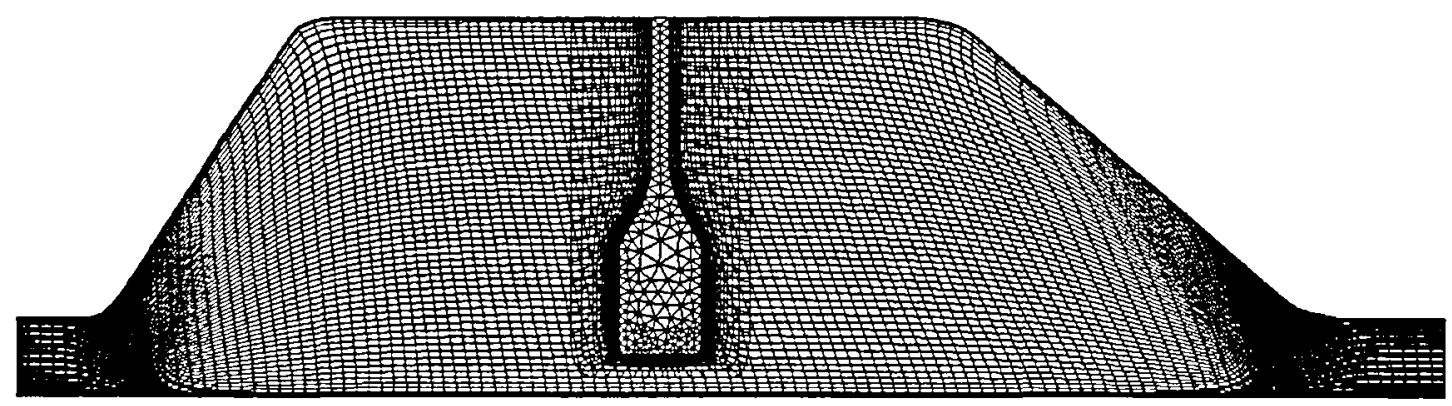

Fig. 12 Simplified drum-disk system using structured/unstructured grids.

(a) adiabatic wall

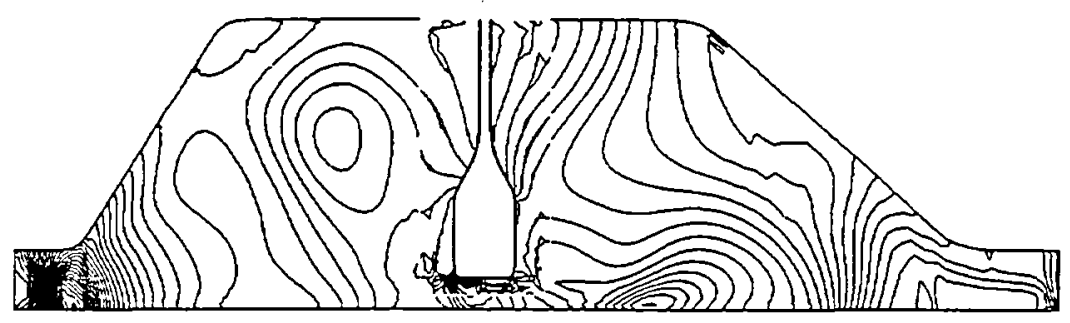

(b) conjugate wall

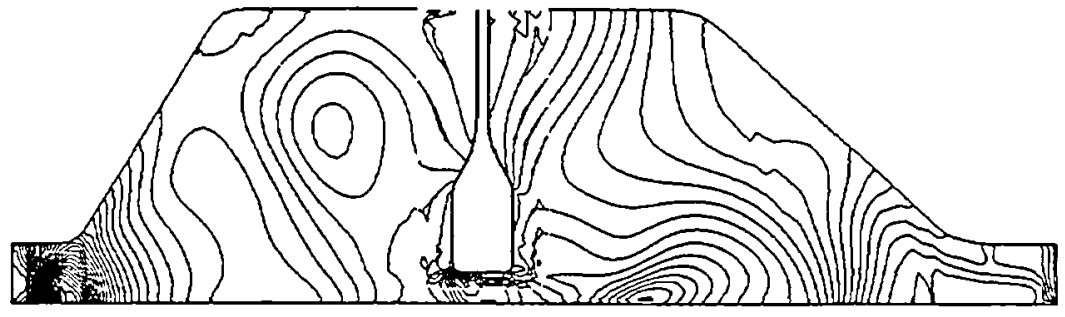

Fig. 13 Comparisons of the pressure contours for simplified drum-disk system.

(a) adiabatic wall, (b) conjugate wall. $\left(\mathrm{P}_{\max }=1.44, \mathrm{P}_{\min }=0.34, \Delta \mathrm{P}=0.026\right)$ 
(a) adiabatic wall

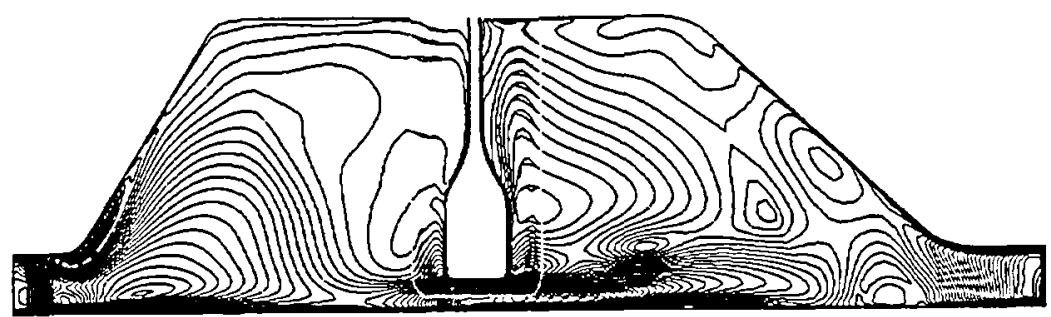

(b) conjugate wall

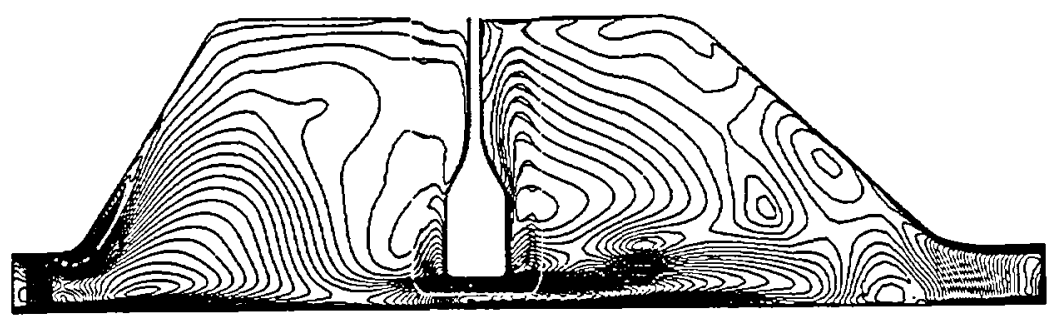

Fig. 14 Comparisons of Mach number contours for the simplified drum-disk system.

(a) adiabatic wall, (b) conjugate wall. $\left(\mathrm{M}_{\max }=0.7, \mathrm{M}_{\min }=0, \Delta \mathrm{M}=0.03\right.$ )

(a) adiabatic wall

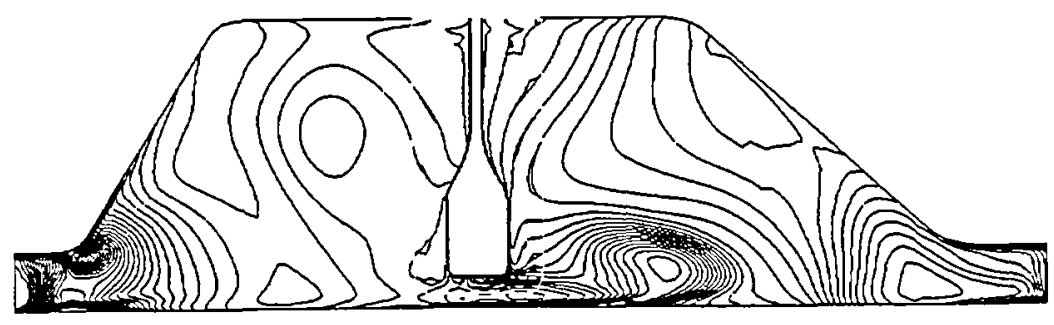

(b) conjugate wall

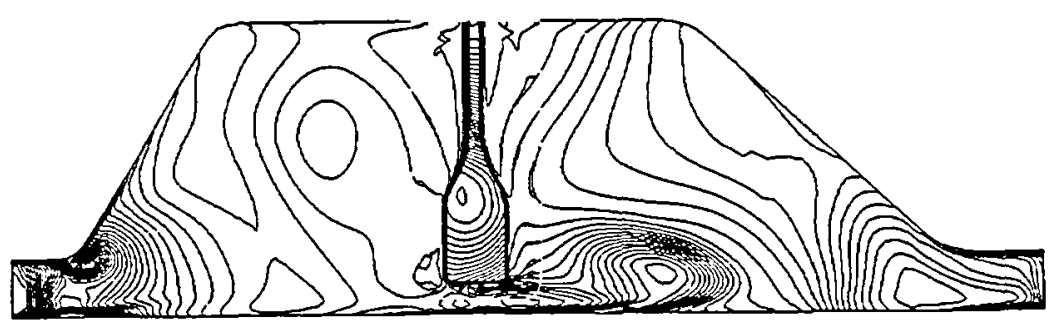

Fig. 15 Comparisons of the temperature contours for the simplified drum-disk system.

(a) adiabatic wall, (b) conjugate wall.

( $\mathrm{T}_{\max }=1, \mathrm{~T}_{\min }=0.6, \Delta \mathrm{T}=0.01$ - fluid region;

$\mathrm{T}_{\max }=0.7, \mathrm{~T}_{\min }=0.6, \Delta \mathrm{T}=0.0004$ - disk region) 


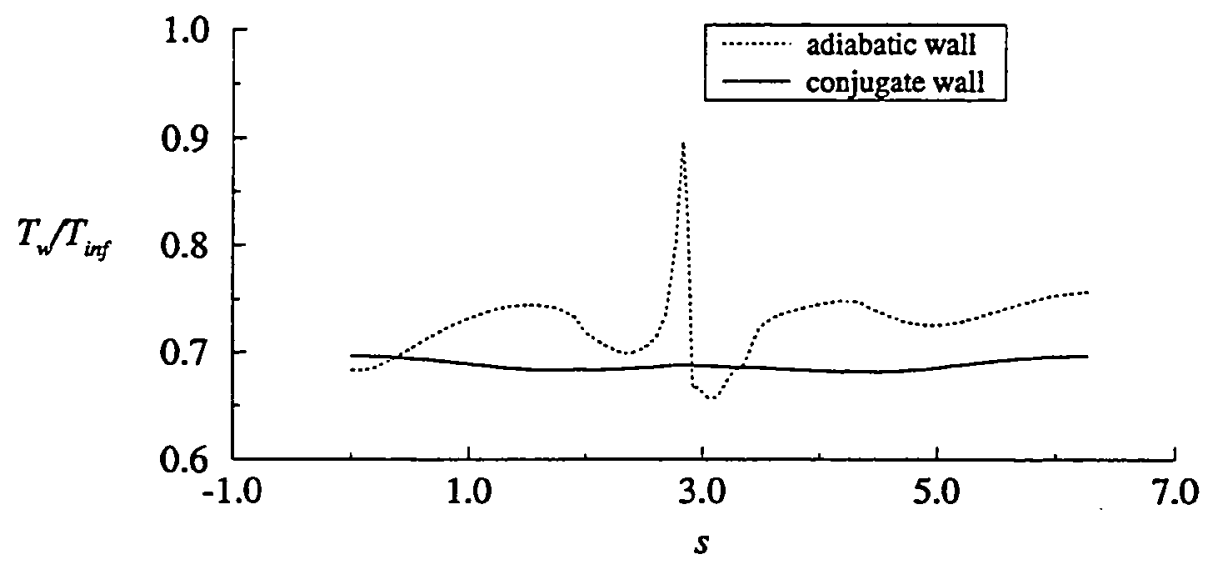

Fig. 16 Temperature distributions along the disk body surface. 
Public reporting burden for this colbction of information ts estimated to average 1 hour per response, including the time for reviewing instructions. searching existing data sources, gathering and maintaining the data needed, and completing and reviowing the collection of information. Send comments regarding this burden estimate or any other aspect of this collection of information, including suggestions for reducing this burden. to Washington Headquarters Services. Directorate for Information Operations and Reports. 1215 Jeflerson Davis Highway, Suite 1204, Arlington, VA 22202-4302, and to the Office of Management and Budget, Paperwork Reduction Project (0704-0183), Washington, DC 20503.

\begin{tabular}{|l|c|c|c|}
\hline 1. AGENCY USE ONLY (Leave blank) & $\begin{array}{c}\text { 2. REPOAT DATE } \\
\text { December 1995 }\end{array}$ & $\begin{array}{r}\text { 3. REPORT TYPE AND DATES COVERED } \\
\text { Technical Memorandum }\end{array}$ \\
\hline
\end{tabular}

4. TITLE AND SUBTITLE

On the Application of Chimera/Unstructured Hybrid Grids for

Conjugate Heat Transfer

6. AUTHOR(S)

Kai-Hsiung Kao and Meng-Sing Liou

\section{FUNDING NUMBERS}

WU-505-90-5K

NCC3-370
7. PERFORMING ORGANIZATION NAME(S) AND ADDRESS(ES)

National Aeronautics and Space Administration

Lewis Research Center

Cleveland, Ohio 44135-3191
8. PERforming organization REPORT NUMBER

E-10035
9. SPONSORING/MONITORING AGENCY NAME(S) AND ADDRESS(ES)

National Aeronautics and Space Administration

Washington, D.C. 20546-0001
10. SPONSORING/MONITORING AGENCY REPORT NUMBER

NASA TM-107121

ICOMP-95-27

11. SUPPLEMENTARY NOTES

Prepared for the ASME Turbo Expo' 96, Land, Sea, and Air, sponsored by the American Society of Mechanical Engineers, Birmingham,

England, United Kingdom, June 10-13, 1996. Kai-Hsiung Kao, Institute for Computational Mechanics in Propulsion, NASA Lewis

Research Center, (work funded under NASA Cooperative Agreement NCC3-233); Meng-Sing Liou, NASA Lewis Research Center. ICOMP Program Director, Louis A. Povinelli, organization code 2600, (216) 433-5818.

12a. DISTRIBUTIONVAVAILABILTY STATEMENT

Unclassified - Unlimited

Subject Category 64

This publication is available from the NASA Center for Aerospace Information, (301) 621-0390.

13. ABSTRACT (Maximum 200 words)

A hybrid grid system that combines the Chimera overset grid scheme and an unstructured grid method is developed to study fluid flow and heat transfer problems. With the proposed method, the solid structural region, in which only the heat conduction is considered, can be easily represented using an unstructured grid method. As for the fluid flow region external to the solid material, the Chimera overset grid scheme has been shown to be very flexible and efficient in resolving complex configurations. The numerical analyses require the flow field solution and material thermal response to be obtained simultaneously. A continuous transfer of temperature and heat flux is specified at the interface, which connects the solid structure and the fluid flow as and integral system. Numerical results are compared with analytical and experimental data for a flat plate and a C3X cooled turbine cascade. A simplified drum-disk system is also simulated to show the effectiveness of this hybrid grid system.

Chimera overset grid; Hybrid grids; Conjugate heat transfer

\begin{tabular}{|c|c|}
\hline 17. SECURITY CLASSIFICATION & 18. SECURITY CLASSIFICATION \\
OF REPORT & OF THIS PAGE \\
Unclassified & Unclassified \\
\hline
\end{tabular}

19. SECURITY CLASSIFICATION OF ABSTRACT Unclassified 
National Aeronautics and

Space Administration

\section{Lewis Research Center}

Cleveland, $\mathrm{OH}$ 44135-3191

ICOMP OAI

Otficlal Business

Penalty for Privale Use $\$ 300$

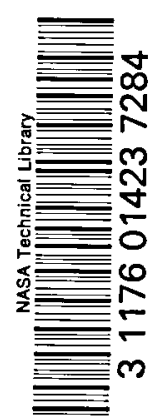

